
\title{
Reseacch S Suare \\ Synthesis, Biological Evaluation and Quantum Chemical Studies of novel 3-Acetylpyridin-2(1H)-one Derivative
}

Hiba H. Ibraheem Al-Mosauy ( $\sim$ Hiba.H.lbraheem@uotechnology.edu.iq )

UOT https://orcid.org/0000-0002-6870-9249

Yasameen Kadhim Al-Majedy

Ali Abdullah Issa

\section{Research Article}

Keywords: DFT, chalcone, pyridine, 2H-chromen-2-one, antifungal, 2H-chromen-2-one, Antioxidant.

Posted Date: February 25th, 2022

DOI: https://doi.org/10.21203/rs.3.rs-1376918/v1

License: (c) (i) This work is licensed under a Creative Commons Attribution 4.0 International License.

Read Full License 


\section{Abstract}

2, 4-dihydroxybenzaldehyde reacts for ethyl acetoacetate yielded 3-acetyl-7-hydroxy-2H-chromen-2-one (1), which was then cyclized with ethyl acetoacetate in the presence of ammonium acetate to give compound 3-acetyl-4-(4-(dimethylamino) phenyl) -6-(7-hydroxy-2-oxo-2H-chromen-3-yl)pyridin-2(1H)-one (2). Melting point, TLC, FT-IR spectroscopy, and ${ }^{1} \mathrm{H}-\mathrm{NMR}$ were used to characterize the newly synthesized compound. Pyridine (2) is a synthetic compound. Staphylococcus aureus, Staphylococcus epidermidis, Escherichia coli, Klebsiella sp., and Candida albicans were chosen for their antibacterial and antifungal properties. Compounds 2 demonstrated strong antibacterial activity when compared to the regular drug Ampicillin using the diffusion process. Compound 2 showed high antifungal efficacy as compared to the standard medication fluconazole. The results demonstrated that the tested compounds 1 and 2 have substantially stronger antioxidant activity than the well-known antioxidant ascorbic acid. They had a high ability for scavenging DPPH and hydroxyl radicals. Quantum chemical parameters for the highest occupied molecular orbital energy (EHOMO) and the lowest unoccupied molecular orbital energy (ELUMO) were determined and presented.

\section{Introduction}

Antibiotic resistance has been on the rise. Because of the serious health threat posed by bacterial and fungal resistance, as well as the rapid spread of the disease [1]. Antifungal medications are used to treat athlete's foot, ringworm, candidiasis (thrust), and severe systemic infections including cryptococcal meningitis [2]. Dermatophytic fungi and yeasts cause onychomycosis, a fungal infection of the nails. Trichophyton, Epidermophyton, and Candida fungi are the major etiological agents. 6.7 Onychomycosis is a rapidly spreading disease that affects people all over the world, it accounts for 50 percent of nail disorders $1,8,9$ and affects $2-9 \%$ of the global population. 2,10This pathological disorder has a negative impact on the patient's quality of life, resulting in a loss of self-esteem and physical, occupational, and social limitations. [3, 4].

Pyridine is used as a pharmacophore for agrochemicals because it is an essential aromatic heterocyclic organic solvent and reagent [5]. Pyridine and its derivatives have been added to a variety of polysaccharides to enhance mental absorption, gene carriers, antimicrobials, sensors, and biomedical applications may all benefit from their solubility, physicochemical properties, and biological properties [6, 7]. Pyridine is a commonly used N-heteroaromatic that can be found in a variety of pharmaceuticals. Antihypertensive properties have been discovered in cyanopyridines (nicotinonitriles) with different alkyl and aryl groups [8], anti-inflammatory, analgesic, antipyretic, and antimicrobial properties $[9,10]$.

Over the last decade, drug resistance has become an increasing problem in the treatment of infectious diseases caused by bacteria, fungi, and viruses. Bacterial infection resistance to currently available antibiotics has emerged as a major public health problem. These active pharmacophores, it has been discovered, If they're connected together, they'll make new molecular templates with fascinating biological properties in animal models. The aforementioned applications prompted us to develop a series 
of new compounds, which we describe in this article. We concentrate on developing a method Using conjugated systems and electron density to improve inhibitive properties, as well as theoretical studies to relate inhibitive properties to electronic structures. For the synthesis of 3-acetyl-4-(4-(dimethylamino) phenyl) -6-(7-hydroxy-2-oxo-2H-chromen-3-yl) pyridin-2(1H)-one (ACP) (Fig. 1), we continued with 3acetylcoumarin derivative as a starting point for a project.

\section{Experimental}

\subsection{General}

The melting points were calculated in an open capillary tube and are unadjusted. On a Perkin-Elmer 157 infrared spectrometer, IR spectra (in $\mathrm{cm}^{-1}$ ) were registered in $\mathrm{KBr}^{1}{ }^{1} \mathrm{H}-\mathrm{NMR}$ ON AMR 400 using DMSO-d 6 solvent, TMS as internal standard. Compound were checked for their homogeneity by TLC on silica gel G plates using hexane and ethyl acetate $(\mathrm{v} / \mathrm{v})$ of varying polarities and spots were visualized by iodine vapor.

\subsection{3-acetylcoumarin derivative (1):}

In ethanol (20 ml. ), a mixture of 2,4-dihydroxybenzaldehyde (1.38 gm.,0.01 mole), piperidine (1.00 ml. ), and ethyl acetoacetate $(0.01$ mole) is refluxed for 8 hours. The progress of the reaction was monitored by TLC (hexane: ethylacetate, 7:3, Rf $=0.62$ for compound (1)). When poured onto an ice-water mixture containing a few drops of hydrochloric acid, solid products form, the resulting solid is filtered off and recrystallized from ethanol M.P $(221-223){ }^{\circ} \mathrm{C}$, brawn precipitate, yield 80 percent Assimilation was seen in the FTIR spectral information (Fig. 2) for (1720 $\mathrm{cm}^{-1}$, belonging to $\cup \mathrm{C}=0$, ketone), $\left(1662 \mathrm{~cm}^{-1}\right.$, belonging to $\cup C=0$, coumarin), $\left(1616 \mathrm{~cm}-1\right.$, for $\cup C=C$, Ar.), $(3049 \mathrm{~cm}-1$, for $\cup C-H, A r),.\left(2991 \mathrm{~cm}^{-1}\right.$, for $\cup C-$ $\mathrm{H}$, aleph.) and $\left(3086 \mathrm{~cm}^{-1}\right.$, for $\left.\mathrm{U}-\mathrm{OH}\right)$. Information from ${ }^{1} \mathrm{H}-\mathrm{NMR}$ spectra revealed a signal inside. 2.3 $\left(\mathrm{s}, 3 \mathrm{H}, \mathrm{CH}_{3}\right), 6.7-8.5(\mathrm{~m}, 4 \mathrm{H}, \mathrm{Ar}-\mathrm{H}$ and $\mathrm{C}=\mathrm{CH})$ and $10(\mathrm{~s}, 1 \mathrm{H},-\mathrm{OH})$. The physical properties of this compound are listed in Table (1).

\subsection{3-acetyl-4-(4-(dimethylamino)phenyl)-6-(7-hydroxy-2- oxo-2H-chromen-3-yl)pyridin-2(1H)-one (2)}

A solution of 3-acetyl-7-hydroxy-2H-chromen-2-one (1) (0.001 mole) in ethanol (10 ml.), 4-N, Ndimethylaminobenzaldehyde ( 0.001 mole), ethyl acetoacetate ( 0.001 mole), ammonium acetate (0.6g.,0.008 mole) were added [11, 12]. For 2 hours, the reaction mixture was refluxed. TLC was used to ensure that the reaction was completed and that the compound was pure (mobile phase hexane: methyl acetate 7:3). After that, the solid was filtered, washed in water, dried, and recrystallized from ethanol. M.P $(273-275){ }^{\circ} \mathrm{C}$, dark orange precipitate, yield $75 \%$. Assimilation was seen in the FTIR spectral data (Fig. 3) for $\left(1726 \mathrm{~cm}^{-1}\right.$, belonging to $\cup C=0$, ketone), $\left(1701 \mathrm{~cm}^{-1}\right.$, belonging to $\cup C=0$, coumarin $),\left(1645 \mathrm{~cm}^{-1}\right.$, belonging to $\cup C=0$, amide), (1597 and $1444 \mathrm{~cm}-1$, for $\cup C=C$, Ar.), $\left(3091 \mathrm{~cm}^{-1}\right.$, for $\cup C-H, A r$.), (2897 and $2814 \mathrm{~cm}^{-1}$, for $\mathrm{UC}-\mathrm{H}$, alph.) and $\left(3225 \mathrm{~cm}^{-1}\right.$, for $\left.\mathrm{U}-\mathrm{OH}\right)$. Signal information from ${ }^{1} \mathrm{H}-\mathrm{NMR}$ spectra was 
shown within (Fig. 4): $1.9\left(\mathrm{~s}, 6 \mathrm{H}, 2 \mathrm{CH}_{3}\right), 2.9\left(\mathrm{~s}, 3 \mathrm{H}, \mathrm{CH}_{3}\right), 7,2-7.8(\mathrm{~m}, 9 \mathrm{H}, \mathrm{Ar}-\mathrm{H}$ and $\mathrm{C}=\mathrm{CH}), 8.5(\mathrm{~s}, 1 \mathrm{H},-\mathrm{OH})$ and $10.00(\mathrm{~s}, 1 \mathrm{H},-\mathrm{NH}) .$. The physical properties of compound (2) are listed in table (1).

\subsection{Evaluation of Antibacterial and Antifungal Assay}

According to the diffusion process, the synthesized compounds were tested for antifungal activity against Candida albicans, and antibacterial gram-positive Staphylococcus aureus, Streptococcus epidermidis, and gram-negative E. coli, Klebsiella sp. [15] at a concentration of $10 \mu \mathrm{g} / \mathrm{L}$ and $1 \mu \mathrm{g} / \mathrm{L}$ Ampicillin and Fluconazole were used as standards for antibacterial and antifungal activity respectively.

\subsection{Antioxidant Activity}

\subsubsection{DPPH radical scavenging activity assay.}

The DPPH radical scavenging activity experiment [16] was done with minimal changes. Antioxidant capabilities of synthesized compounds ( 1 and 2) were examined spectrophotometrically using 2,2diphenyl-1-picrylhydrazyl radical.Various quantities of the produced compounds (250, 500, 750 and 1000 $\mathrm{mg} / \mathrm{mL}$ ) were utilized. $6.0 \mathrm{mg}$ of DPPH was dissolved in $100 \mathrm{~mL}$ of the solvent to make the DPPH solution (methanol). Then, $1 \mathrm{~mL}$ of each concentration was added to $2 \mathrm{~mL}$ of the DPPH solution that had been created. Finally, a control was made by combining $1 \mathrm{~mL}$ methanol with $2 \mathrm{~mL}$ DPPH. As a benchmark, Trolox was used. The mixture was violently mixed before being incubated in the dark for 30 minutes. At $517 \mathrm{~nm}$, the absorbances of the resultant solutions were measured spectrophotometrically. The scavenging activities of each prepared concentration of the DPPH radicals were estimated using Eq. (1).

$\mathrm{DPPH}_{\text {scavenginig effect }} \%=\left(\mathrm{A}^{\circ}-\mathrm{A} / \mathrm{A}^{\circ}\right) * 100$

where $A^{\circ}$ is the absorbance of the control reaction and $A$ is the absorbance when samples or standards are present. In triplicate, all samples were produced and quantified.

\subsubsection{Hydrogen Peroxide Scavenging Activity}

In phosphate buffer, a $40 \mathrm{mM}$ hydrogen peroxide solution was produced ( $\mathrm{pH}$ 7.4). The synthesized compounds (or ascorbic acid) were added to a hydrogen peroxide solution at various concentrations $\left(250,500,750\right.$, and $\left.1000 \mathrm{~g} \mathrm{~mL}^{-1}\right)(0.6 \mathrm{~mL}, 40 \mathrm{mM})$. After 10 minutes, the absorbance of hydrogen peroxide at $230 \mathrm{~nm}$ was measured against a blank solution of phosphate buffer without hydrogen peroxide [17]. The hydrogen peroxide percentage scavenging activity was calculated using Eq. 1.

\subsection{Computational method}

The Gaussian 09, revision A.02 software was used to perform all theoretical calculations on the compounds studied and the Lee-Yang-Parr (LYP) and the correlation functional, as well as Becke's hybrid three-parameter in combination (B3)interchange functional (B3LYP) The basis set and a density function 
theory-based approach (DFT), (6-311G (To measure both largest occupied molecular orbital energies $\left(\mathrm{E}_{\mathrm{HOMO}}\right)$, lowest unoccupied molecular orbital energies $\left.\left(\mathrm{E}_{\mathrm{LUMO}}\right)\right][18[$.

\section{Results And Discussion}

\subsection{Chemistry}

Using the cyclization of (3-acetyl-7-hydroxy-2H-chromen-2-one), comp 1, the starting material (3-acetyl-7hydroxy-2H-chromen-2-one) was originally prepared by condensing 2,4-dihydroxybenzaldehyd with ethyl acetoacetate acid in the presence of piperidine [17], (3-acetyl-4-(4-(dimethylamino) phenyl)-6-(7-hydroxy-2oxo-2H-chromen-3-yl) pyridin-2(1H)-one) comp (2). with ethyl acetoacetate acid in ethanol as illustrated in Figure (1), was prepared with good yields. The absorption bands in the IR spectra of pyridine 2 were found at $1726 \mathrm{~cm}-1(\mathrm{C}=0$, ketone) and $2897,2814 \mathrm{~cm}-1$ (alephatic $\mathrm{C}-\mathrm{H})$, respectively. The $-\mathrm{NH}$ proton at $10.5 \mathrm{ppm}$ caused a singlet in the $1 \mathrm{H}-\mathrm{NMR}$ spectra. At $2.3 \mathrm{ppm}$, methyl protons resonant as a singlet.

\subsection{Antibacterial activity}

The antibacterial activity of compounds (1) and (2) identified here was investigated. Good results were obtained in the cases of (3-acetyl-4-(4-(dimethylamino) phenyl)-6-(7-hydroxy-2-oxo-2H-chromen-3-yl) pyridin-2(1H)-one) comp (2). This compound was found to possess high Antibacterial activity against Staphyloccocus aureus and were slightly less active against Klebsiella sp.(Table 2) The introduction of pyridine ring was much more important in increasing antifungal activity. Based on the above-mentioned findings, compound (2) enhanced the antifungal activity compared with 3-acetyl-7-hydroxy-2H-chromen-

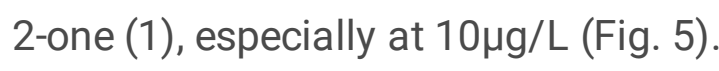

Table 1

Some physical properties of the prepared compounds

\begin{tabular}{|lllllll|}
\hline No. & M.F & M.W & M.P ${ }^{\circ} \mathrm{C}$ & Yield & $\begin{array}{l}\text { Rf(7:3) } \\
\text { Hexane.: Eth.acetate }\end{array}$ & Color \\
\hline 1 & $\mathrm{C}_{11} \mathrm{H}_{8} \mathrm{O}_{4}$ & 204 & $244-246$ & 82 & 0.67 & brawn \\
\hline 2 & $\mathrm{C}_{24} \mathrm{H}_{20} \mathrm{~N}_{2} \mathrm{O}_{5}$ & 416 & $253-275$ & 75 & 0.69 & Dark orange \\
\hline
\end{tabular}


Table 2

Antimicrobial Activity of the Synthesized compounds (1 and 2).

\begin{tabular}{|c|c|c|c|c|c|c|c|c|}
\hline \multirow{3}{*}{$\begin{array}{l}\text { Comp. } \\
\text { No. }\end{array}$} & \multicolumn{4}{|c|}{ Gram positive } & \multicolumn{4}{|c|}{ Gram negative } \\
\hline & \multicolumn{2}{|c|}{ S. aureus } & \multicolumn{2}{|c|}{ Streptococcus epidermidis } & \multicolumn{2}{|l|}{ E. coli } & \multicolumn{2}{|c|}{ Klebsiella sp. } \\
\hline & $10 \mu \mathrm{g} / \mathrm{L}$ & $1 \mu \mathrm{g} / \mathrm{L}$ & $10 \mu \mathrm{g} / \mathrm{L}$ & $1 \mu \mathrm{g} / \mathrm{L}$ & $10 \mu g / L$ & $1 \mu \mathrm{g} / \mathrm{L}$ & $10 \mu \mathrm{g} / \mathrm{L}$ & $1 \mu \mathrm{g} / \mathrm{L}$ \\
\hline 1 & 14 & 12 & - & - & - & - & - & - \\
\hline 2 & 18 & 16 & 12 & 10 & 12 & 10 & 12 & 10 \\
\hline Amp. & 14 & 12 & 14 & 12 & 14 & 12 & 14 & 12 \\
\hline control & - & - & - & - & - & - & - & - \\
\hline
\end{tabular}

The addition of the pyridine ring improved the antifungal activity significantly. Because of its lipophilic nature, outside the cell membranes, the heterocyclic aromatic ring is more likely to move through the oil film, causing cell membrane damage [19].

\subsection{Antifungal activity}

Tow from the (1), and (2) compounds were identified.These prepared compound (2) showed antifungal activity in comparison with the antifungal drug utilized (fluconazole), which was used as a standard (Fig. 6). While other compound showed moderate effectiveness against fungi till minimal antifungal activity [20]. Despite the explanations for the organic compounds, its effectiveness and inefficiency against fungi are not understood, but the results can be explained by the appearance and non-emergence of activity against fungi as a whole.

- Interference with the synthesis of cellular walls, causing damage that can lead to altered cell permeability characteristics or disorganized lipoprotein arrangements, ultimately resulting in cell death.

- Delocalization of $\pi$-electrons over the compounds (1) and (2) increases lipophilicity which in turn facilitates the penetration of the compounds (1) and (2) into lipid membranes, further restricting proliferation of the microorganisms.

- The electron donating dimethyl amine group of phenyl ring through compund (2) It is an effective activity against fungi and becteria.

- Formation of a hydrogen bond through the hydroxyl group with the active centers of various cellular constituents, resulting in interference with normal cellular processes

\subsection{Antioxidant assay}

\subsubsection{DPPH radical scavenging activity assay.}


The DPPH radical, which stands for 1,1-diphenyl-2-picrylhydrazyl, is a stable radical that can share an electron or hydrogen and transfer it to a stable molecule. The single-electron (ode-electron) in the methanolic DPPH solution showed a significant absorption band at $517 \mathrm{~nm}$, and if this solution was mixed with suitable reducing agents, the consequence would be electron pairing and a loss of color. As the quantity of electrons taken up grows, the color is stoichiometrically lowered, and the decrease in absorbance may be directly measured and compared to the standard (ascorbic acid). In comparison to the ascorbic acid standard, Fig. 7 indicates the high DPPH radical scavenging capabilities.

\subsection{2 $\mathrm{H}_{2} \mathrm{O}_{2}$ scavenging activity}

When hydrogen peroxide crosses the cell membrane, it can create the hydroxyl radical, which is very reactive. A Fig. 8 show that compounds 1 and 2 have a high hydrogen peroxide scavenging action at a very low concentration of $250 \mathrm{~g} / \mathrm{mL}$, with a concentration dependant reduction. Compound 1 (27.0 3.00) showed a very mild inhibitory effect. At $1000 \mathrm{~g} / \mathrm{mL}$, the greatest concentration was discovered (Fig. 8). Compound 2 had the highest percentage scavenging activity (73.0 5.00). Standard medicines included ascorbic acid, which had a percentage inhibition of 70.002 .5 .

\subsubsection{Suggested mechanism for compounds 1 and 2 as antioxidants}

The suggested antioxidant mechanism (Figs. 9 and 10) for synthesized copounds (1 and 2), as shown in Figure (9 and 10), relies on the hydrogen atom of hydroxyl (enol form) (for compound 1 and 2) and hydrogen atom of amino group (for compound 2), which were under the influence of resonance and inductive effects. The resonance and inductive effects facilitates the release of hydrogen, resulting in stability of the molecule. [17] Coumarinpyridines have scavenging activities due to the stability of the free radical intermediates of these compounds. An abstraction of a hydrogen atom from hydroxyl (enol form) and amine group may occur easily. The presence of carbonyl groups, enhances the antioxidant activity. The steric hindrance enhances the antioxidant activity.

\subsection{Theory of Density Functions (DFT)}

Compounds 1 and 2 were subjected to DFT measurements. Figures 11 and 12 depicts optimized molecular structures of the most stable types. Table 3 shows the measured and relative energies of the participants. Theoretical findings using the measurement method proposed in this study revealed that compound 2 is more stable than other compounds based on the following factors: total energy, and higher energy orbital molecular occupied $\left(\mathrm{E}_{\mathrm{HOMO}}\right)$, Calculations of molecular orbitals provide a comprehensive overview including their spatial properties, of orbitals. Private atom contributions and nodal trends The contour plots of the frontier orbitals for ground states 1 and 2 are shown in Figs. 12 and 13, including "the Highest Occupied Molecular Orbital" (HOMO) and "the Lowest Unoccupied Molecular Orbital” (LUMO). 
Table 3

Total Energy (a .u), HOMO and LUMO, HOMO - 1 and LUMO +1 energies of Comp. 1 and 2 (eV).

\begin{tabular}{|llllllll|}
\hline Molecular & Total energy & HOMO & LUMO & $\Delta \mathrm{E}$ & HOMO-1 & LUMO +1 & $\Delta \mathrm{E}$ \\
\hline Comp.1 & $724.674825815-$ & -6.6502 & -2.6403 & 4.0099 & -6.9876 & -0.9934 & 5.9942 \\
\hline Comp.2 & $1411.83996104-$ & -5.0771 & -2.8264 & 2.2507 & -5.6452 & -1.5363 & 4.1089 \\
\hline
\end{tabular}

It's worth noting how equally both orbitals are distributed around the conjugation plane. Figures $3 \& 4$ show that the substituted molecule has HOMO orbitals, whilst the LUMO orbitals are identical to those of the unsubstituted molecule, meaning that the substitution affects the electron donation potential. The ability to accept electrons, on the other hand, is just slightly harmed. Table 3 Compounds 1 and 2's the orbital energy levels of HOMO and LUMO are stated. The disparities in energy between HOMO and LUMO are approximately 4.0099, 2.2507ev. In the case of compound 1, and 2, respectively. Compound 2 also describes the lower the HOMO, LUMO, and energy gap values are, the better. which describes the reaction of final shipping transport within the molecules.

\subsubsection{Atomic Stabilities and Charges}

According to theoretical research, the existence of the ring substituent in compound 1 affected the atomic charges. Figure 3 shows the compound's reduced geometry as well as the measured atomic charges. The maximum atomic charge is shown in Fig. 1. [0 (10) 0.748], and the second highest charge is at [0 (11) 0.769]. These results clearly demonstrate that in terms of reactions and metal bonding, these two atoms are the most reactive. Angles of bonding and twisting that have been measured (supplementary file) this molecule is not planar, according to the optimized structure. Figure 5 depicts the optimized geometry for compound 2. In addition, the diagram shows the calculated atomic charges for the compound. The maximum atomic charge was found at [N (42) 1.104] according to the findings. And [N (24) 0.925] is the next charge value. These results showed that in terms of reactions and metal bonding, these two atoms are the most reactive.

\subsubsection{Geometrical optimization}

In order to understand the molecular properties of the synthesized compounds (1) and (2), we carried out their structural optimization and the energies of the Highly Occupied Molecular Orbital (HOMO) and Least Unoccupied Molecular Orbital (LUMO) were evaluated from the optimized structures. Further, the energies of HOMO and LUMO were utilized to calculate some of the parameters like electronegativity $(X)$, chemical potential $(a)$, hardness $(\eta)$, electrophilicity index $(\omega)$, electron affinity $(I)$ and ionization potential $(A)$ to understand the reactivity of the studied molecules. The global parameters have been calculated by using the following equations.

Ionization potential (I) is related to the energy of the $\mathrm{E}_{\mathrm{HOMO}}$ through the equation [21]:

$\mathrm{I}=-\mathrm{E}_{\mathrm{HOMO}}$-........(2) 
Electron affinity $(A)$ is [21] related to $E_{\text {LUMO }}$ through the equation:

$A=-E_{\text {LUMO }}$

When the values of I and $A$ are known, one can determine the electronegativity $\chi$ and the global hardness

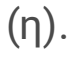

The electronegativity [22], can be estimated by using the equation:

$\chi=\frac{I+A}{2}$

Chemical hardness ( $\eta$ ) measures the resistance of an atom to charge transfer [22], it is estimated by using the equation:

$\eta=\frac{I-A}{2}$

Chemical softness (S), the reverse of hardness [22], is estimated by using the equation:

$\mathrm{S}=\frac{1}{\eta}$

electrophilicity index $(\omega)$ as follows.

$\omega=\frac{\mu^{2}}{2 \eta}$.

Where $\mu$ is the electronic chemical potential [23]:

$\mu=-\chi$

The calculated HOMO-LUMO energies and global parameters of the synthesis compounds (1) and (2) are displayed in Table 3.

The tabulated data reveals that, low energy gap, chemical hardness and electrophilicity index values are responsible for the good biological activity of the pyridine derivative (2) [24, 25].

The optimized molecular structures and HOMO-LUMO energy level diagrams of the synthesis compounds (1) and (2) were shown in Figs. 11, 12, 13, and 14 and Table 4 . The reactivity of molecules often decided by using density functional theory (DFT) and that is based on the energy differences between the HOMO and LUMO. From the literature review, it was observed that if the difference between the HOMO and LUMO is small, the energy required to excite an electron to higher energy state is less and therefore the molecules become more reactive chemically and biologically $[26,27]$. 
Table 4

The quantum chemical parameters evaluated for the synthesis compounds (1) and (2) by DFT method at B3YLP/6-31G (d, p).

\begin{tabular}{|lll|}
\hline Electronic parameters & $(\mathbf{1})$ & $\mathbf{( 2 )}$ \\
\hline Electronegativity $(\mathrm{X})$ & 4.645 & 3.951 \\
\hline Electron Chemical potential $(\mu)$ & -4.645 & -3.951 \\
\hline Chemical Hardness $(\mathrm{\eta})$ & 2.004 & 1.125 \\
\hline Chemical softness $(\mathrm{S})$ & 0.215 & 0.253 \\
\hline Electrophilicity index $(\omega)$ & 5.383 & 6.937 \\
\hline Ionization potential $(\mathrm{I})$ & 6.650 & 5.077 \\
\hline Electron affinity $(\mathrm{A})$ & 2.640 & 2.826 \\
\hline
\end{tabular}

If the gap is large, then the promotion of electron becomes difficult and requires lot of energy, so that the molecules become more stable towards any reaction. Thus, from the above discussion it is inferred that the theoretical modeling is most useful in the interpretation of chemical reactivity, kinetic stability, polarizability and biological properties of the molecules $[28,29]$.

\section{Conclusion}

Antimicrobial screening data revealed that the synthesized compound (2) had antimicrobial activity against Gram-positive, Gram-negative, and Gram-positive bacteria, as well as fungi (Candidida albicans). According to the findings, the functional substitution nitrogen group on the benzene ring at carbon four selectively enhances or increases pyridine activity inhibition, and the hydroxyl group on the benzene ring at carbon six on the coumarin nucleus appears to be a highly significant factor in affecting the compound's biological activity. The antioxidant activities were evaluated by DPPH and hydrogen peroxide assays, and the results indicated that these compounds have good scavenging activities. The antioxidant mechanisms of the synthesized compounds (1) and (2) were also studied. The theoretical determination of atomic charges, formation heat, and stereochemistry are all factors to consider. The synthesized compounds (1) and (2) revealed that they are not planar.

\section{Declarations}

\section{Acknowledgements}

The author is grateful to the (Department of Applied Science - Chemistry Division, University of Technology, and Al-Mustansiriya University - College of Science's Department of Biology for providing the 
required resources for this study.

\section{References}

1. Al-Amiery, A.A.; Mohammed, A.; Ibrahim, H.; Abbas, A. Study the biological activities of tribulus terrestris extracts. World Acad. Sci. Eng. Technol. 2009, 57, 433-435.

2. Onkol T, Mehtap Gokce Ah, Tosun U, Polat S, Mehmet S, Serin ST (2008) Microwave synthesis and antimicrobial evaluation of 5-Chloro-2(3 h) benzoxazolinone-3- acetyl-2-(p-substituted benzal)hydrazone and 5-Chloro-2(3 h)-benzoxazolinone-3-acetyl-2-(p-substituted acetophenone) hydrazone derivative. Turk J Pharm Sci 5(3):155-166

3. Emam SM, Abd El-salam OH. Real-time PCR: a rapid andsensitive method for diagnosis of dermatophyte inducedonychomycosis, a comparative study. Alexandria Med J.2015:1-8.

4. Imbert JL, Gomez JVG, Escudero RB, Blasco JL. Onicomicosispor levaduras no comunes en diabéticos de un centro desalud. J Semergen. 2016;7:449-457.

5. Jia, R.; Duan, Y.; Fang, Q.;Wang, X.; Huang, J. Pyridine-grafted chitosan derivative as an antifungal agent. Food Chem. 2016, 196, 381-387.

6. Yuvaraja, G.; Venkata Subbaiah, M. Removal of Pb(II) ions by using magnetic chitosan-4-((pyridin2-ylimino)methyl)benzaldehyde Schiff's base. Int. J. Biol. Macromol. 2016, 93, 408-417.

7. Goncalves, F.J.; Kamal, F.; Gaucher, A.; Gil, R.; Bourdreux, F.; Martineau-Corcos, C.; Gurgel, L.V.A.; Gil, L.F.; Prim, D. Synthesis, characterisation and application of pyridine-modified chitosan derivatives for the first non-racemic Cu-catalysed Henry reaction. Carbohydr. Polym. 2018, 181, 1206-1212.

8. Abdel LNA (2005) Synthesis and antidepressant activity of some new coumarin derivatives. Sci Pharm 73: 195-216.

9. Manna F, Chimenti F, Bolasco A, Filippelli A, Palla A, et al. (1992) Antiinflammatory, analgesic and antipyretic 4,6-disubstituted 3-cyanopyridine-2- ones and 3-cyano-2-aminopyridines. Eur J Med Chem 27: 627-632.

10. Manna F, Chimenti F, Bolasco A, Bizzarri B, Filippelli W, et al. (1999) Anti-inflammatory, analgesic and antipyretic 4,6-disubstituted 3-cyano-2- aminopyridines. Eur J Med Chem 34: 245-254.

11. Hiba H. Ibraheem, (2019) New derivative of 1,2-Dihydropyridine-3-Carbonitrile as Corrosion Inhibitor, Materials Science and Engineering, 579: 012052.

12. Y K Al-Majedy, H H Ibraheem, M S Falih and A A Al-Amiery, (2019), New coumain derivatives as corrosion inhibitor, Materials Science and Engineering, 579: 012051.

13. Daw, Z.Y.; EL-Baroty, G.S.; Mahmoud, A.E. Inhibition of Aspergillus parasiticus growth and aflatoxin production by some essential oils. Chem. Mikrobiol. Technol. Lebensm. 1994, 16, 129-135.

14. A. AL.Ahmed, A. H. Abdul Amir and M. Abu Bakar, 2012 Antifungal Activities of New Coumarins, molecules , 17:5713-5723.

15. Ibraheem, H. H.; Al-Majedy, Y. K.; Salim, A. J.; Al- Bayati, R. I.; "Synthesis and Characterization of New 1,2-Dihydropyridine-3-Carbonitrile Compounds with Study of Expected Biological Activity"; 
Al- Nahrain J. Sci. 21(2), 45-50, 2018.

16. Chan E. W. C., Lim Y. Y. and Omar M. 2007. Antioxidant and antibacterial activity of leaves of Etlingera species (Zingiberaceae) in Peninsular Malaysia. Food Chemistry, 104 (4): 1586-1593.

17. Yasameen K. Al-Majedy, Hiba H. Ibraheem, Liblab Sami Jassim and Ahmed A. Al-Amiery, (2019) Antioxidant Activity of Coumarine Compounds, Al-Nahrain Journal of Science, 22:1-8.

18. R. Dennington, T. Keith, J. Millam, GaussView, Version 5, Semichem Inc., Shawnee Mission, KS, 2009. 19.

20. Lijie Wei , Wenqiang Tan , Jingjing Zhang , Yingqi Mi , Fang Dong , Qing Li and Zhanyong Guo, 2019 Polymers, 11 (2), pp 371-382

21. AL.Ahmed A. A., Abdul Amir A. H. and Abu Bakar M., 2012 molecules 17, pp 5713-5723.

22. J.B. Foresman; A Frisch, Exploring Chemistry with Electronic Structure Methods. Gaussian, Inc., Pittsburg, PA (USA), 1995.

23. L. Pauling, The Nature of the Chemical Bond. Cornell University Press, Ithaca, New York; 1960.

24. P Senet, Chem. Phys. Lett. 1997,275, 527-532.

25. RG Parr; RA Donnelly; M Levy and WE Palke, J. Chem. Phys., 1978, 68, 3801-3807.

26. J. George, J.C. Prasana, S. Muthu, T.K. Kuruvilla, S. Savanthi, R.S. saji, Spectroscopic (FT-IR,FTRaman)and Quantum mechanical study on N-(2,6 dimehyl phenyl)-2-\{4-[2hydroxy-3(2methoxyphenoxyl)propyl]piperazin-1yl\}acetamide, J. Mol. Struct. 1171 (2018) 268-278.

27. O. Nagaraja, Yadav D. Bodke, Itte Pushpavathi , S. Ravi Kumar, Synthesis, characterization and biological investigations of potentially bioactive heterocyclic compounds containing 4-hydroxy coumarin, Heliyon 6 (2020) e04245, pp. (1-10).

28. V.D. Vitnik, Z.J. Vitnik, The spectroscopic (FT-IR, FT-Raman, I3C, $1 \mathrm{H}$ NMR and UV) and NBO analyses of 4-bromo-1-(ethoxycarbonyl) piperidine-4-carboxylic acid, Spectrochim. Acta Mol. Biomol. Spectrosc. 138 (2015) 1-12.

29. B. Kosar, C. Albayrak, Spectroscopic investigations and quantum chemical computational study of (E)-4-methoxy-2-[(p-tolylimino) methyl] phenol, Spectrochim. Acta Mol. Biomol. Spectrosc. 78 (2011) 160-167.

30. J. Keshavayya Vinodkumar, I. Pushpavathi, C.T. Keerthikumar, M.R. Maliyappa, B.N. Ravi, Synthesis, characterization, computational and biological studies of nitrothiazole incorporated heterocyclic azo dyes, Struct. Chem. (2020).

\section{Supplemental File}

Supplemental file is not available with this version.

\section{Figures}




\section{Image not available with this version}

Figure 1

This image is not available with this version.

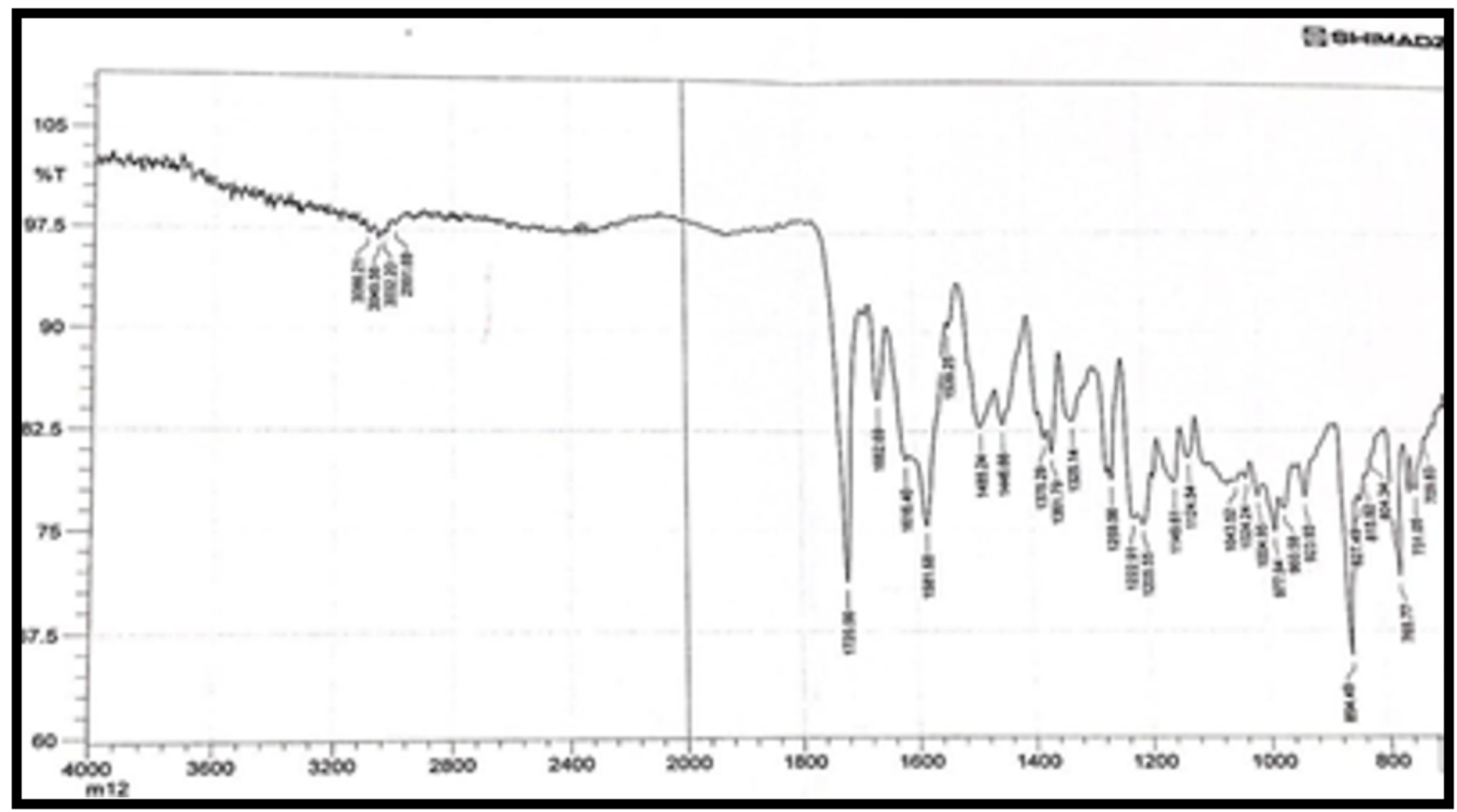

Figure 2

FT-IR curve of 3-acetylcoumarin derivative (1)

Figure 3

FT-IR curve of Pyridine derivative (2). 


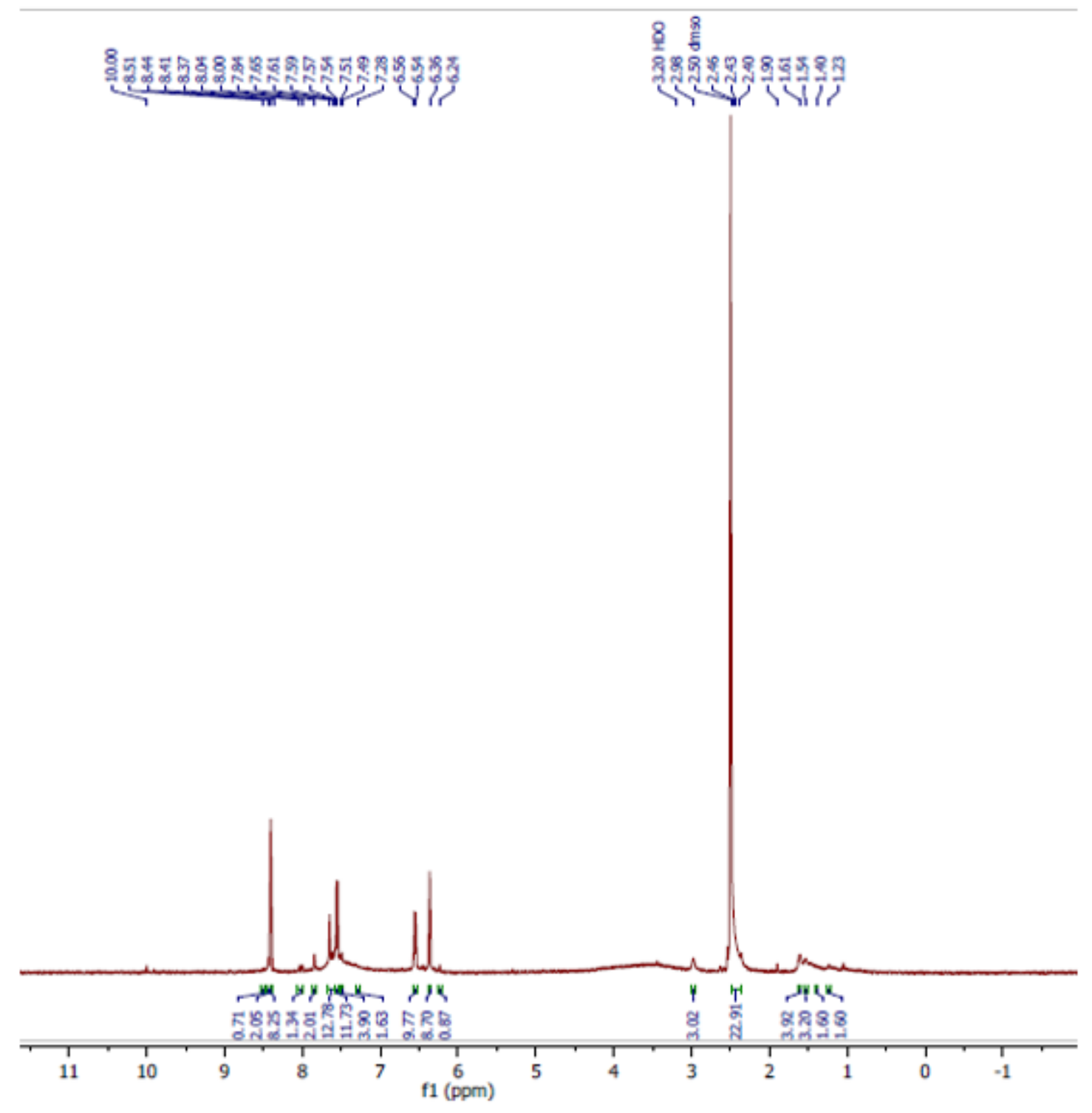

Figure 4

The ${ }^{1} \mathrm{H}-\mathrm{NMR}$ spectrum from Pyridine derivative (2). 

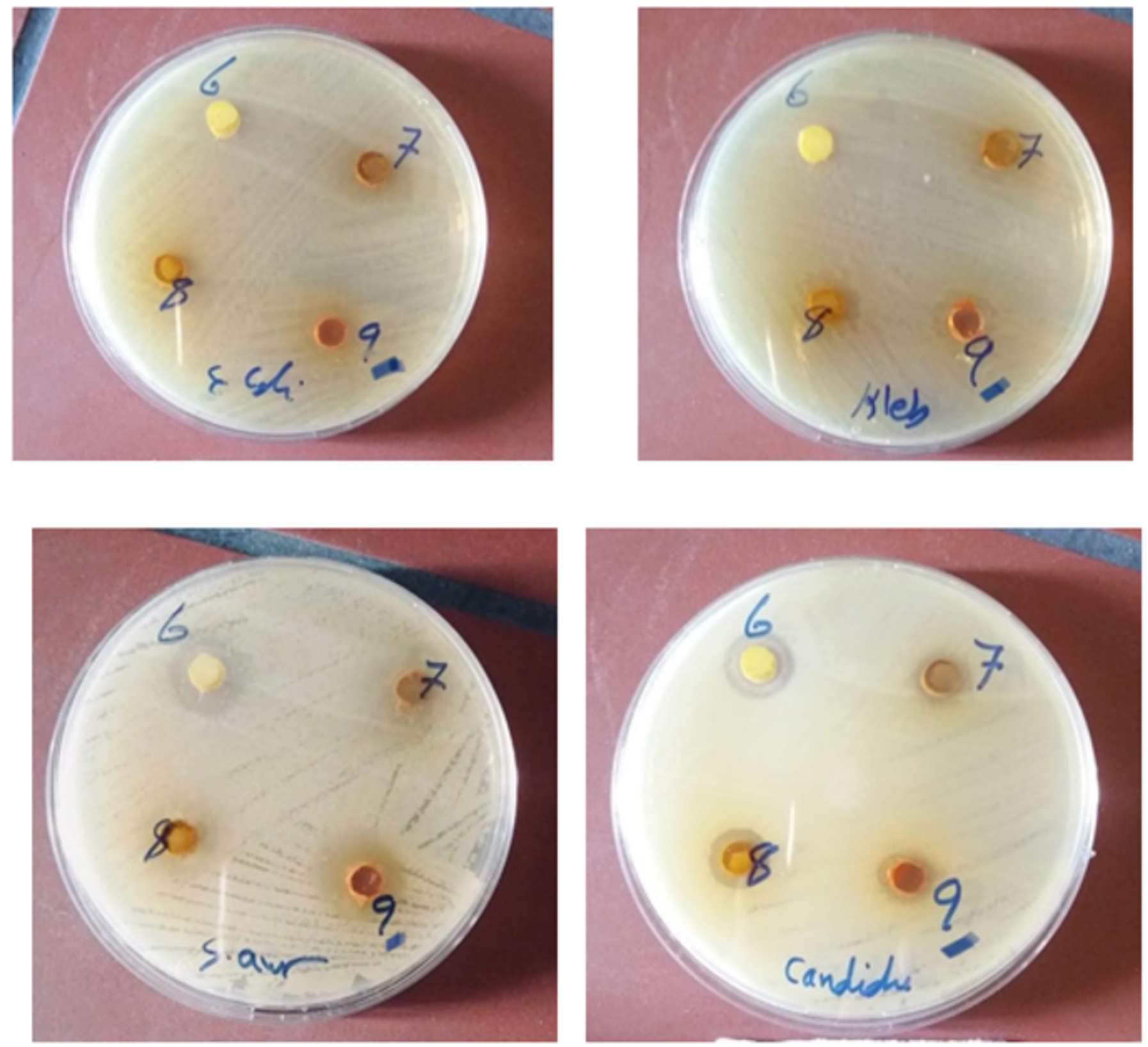

\section{Figure 5}

Inhibition zone of the compound (1) and (2) on the grame positive bacteria (S.aureus), grame negative bacteria (Klebsiella sp., Escherichia coli, ) and Candida albicans at conc. 10 and $1 \mu \mathrm{g} / \mathrm{mL}$ ). 


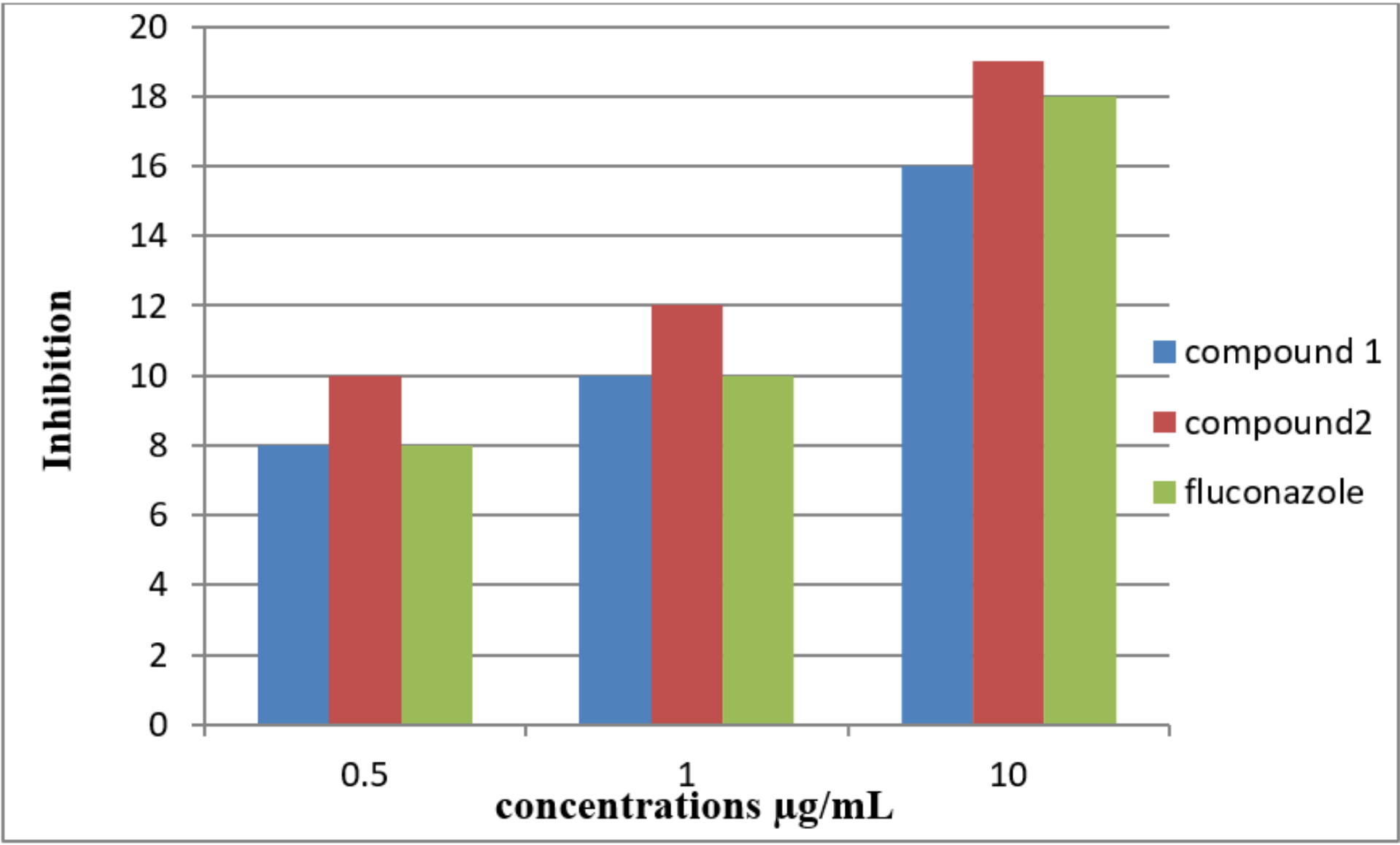

Figure 6

The effect of compounds (1) , (2) and fluconazole toward the tested organism Candida albicans. 


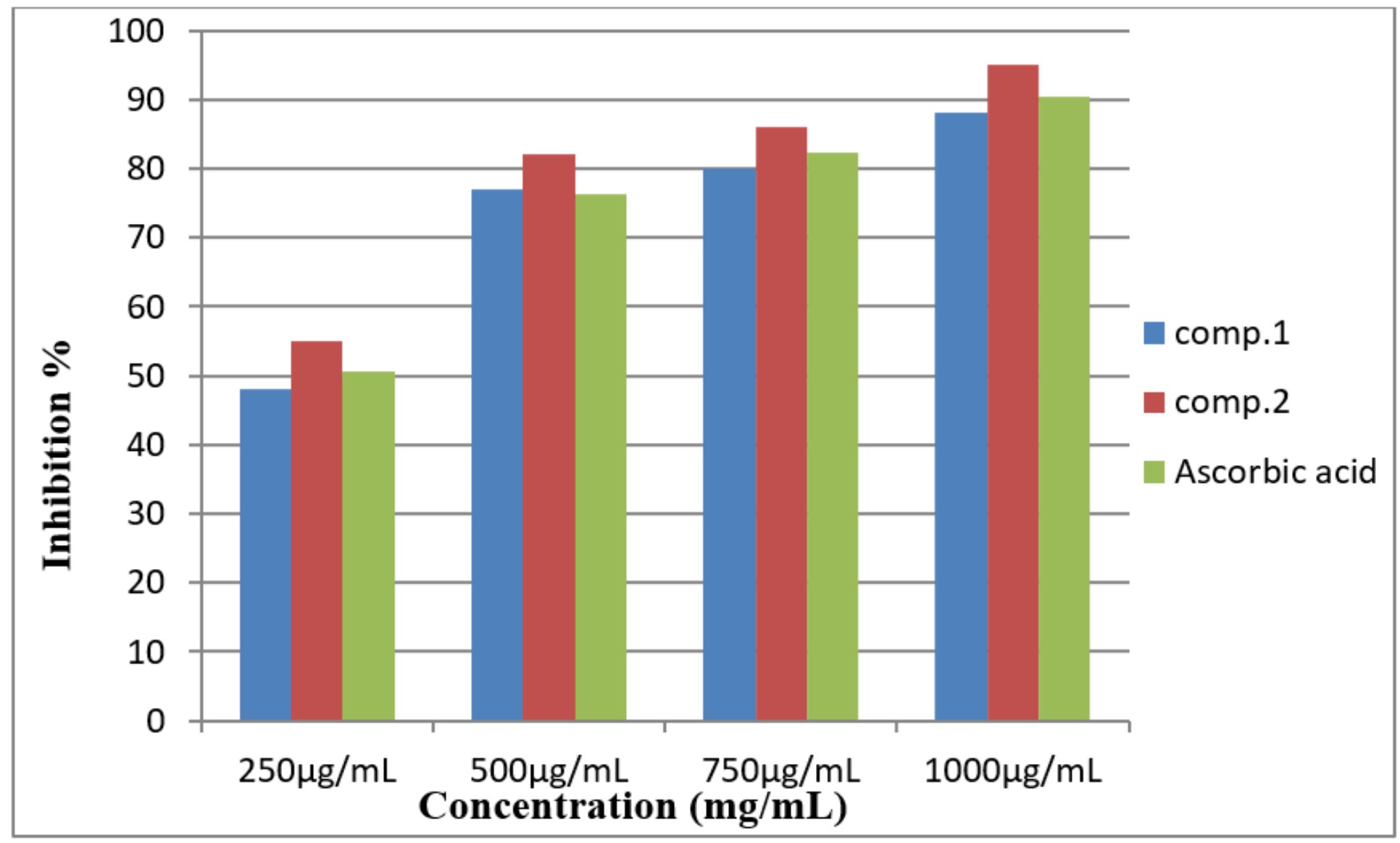

Figure 7

Antioxidant activity of different concentrations of the synthesized compounds ( 1 and 2 ) and ascorbic acid. in the DPPH radical scavenging assay.

Figure 8

Percentage inhibition of hydrogen peroxide scavenging activity of

synthesized compounds $(1,2)$ in comparison to ascorbic acid. $n=3$. Error bars

indicate standard deviation. 


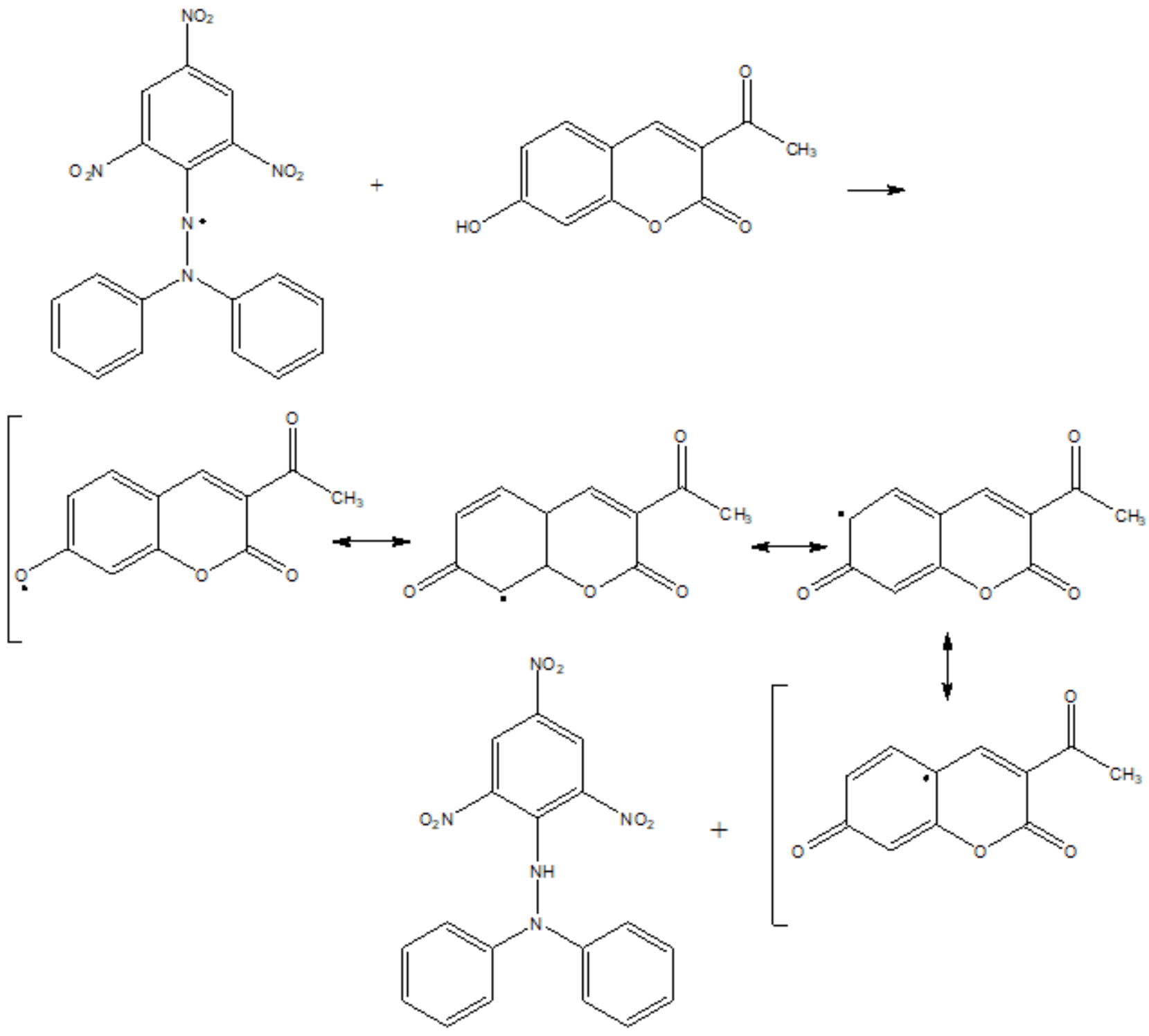

Figure 9

The reaction scheme between DPPH free radicals and compound (1). 
<smiles>CCOc1ccc2cc(-c3cc(-c4ccc(N(C)C)cc4)c(C(C)=O)c(=O)[nH]3)c(=O)oc2c1</smiles>

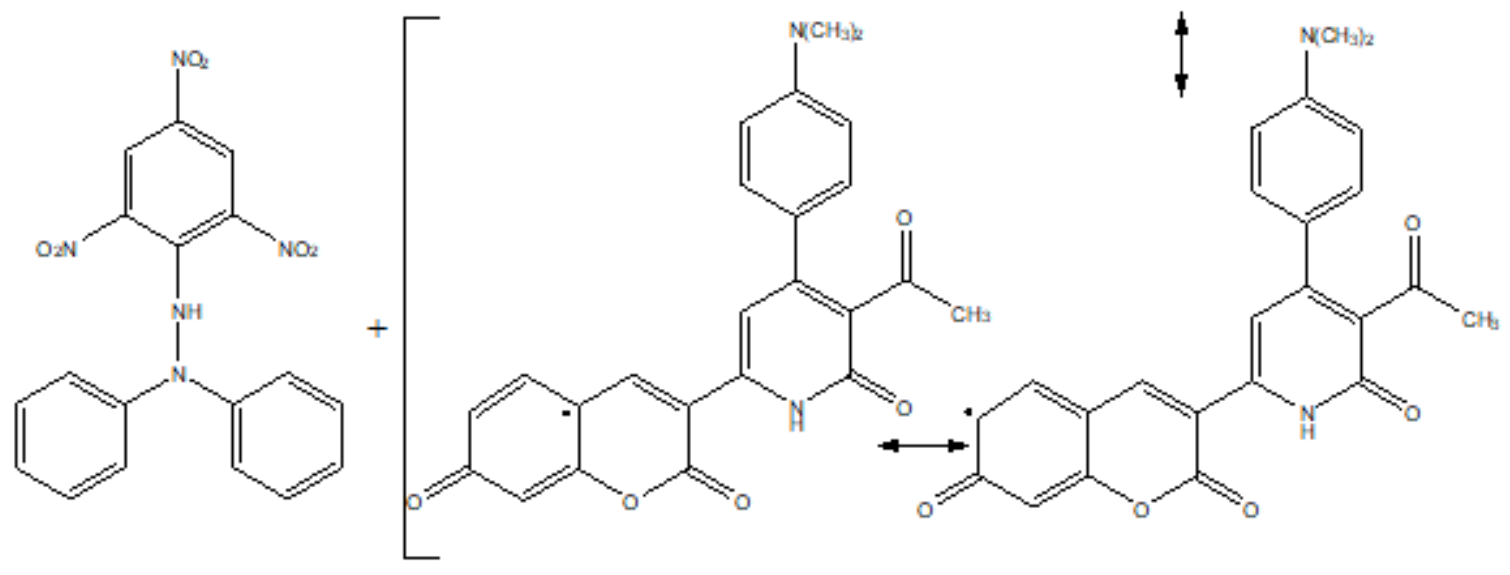

Figure 10

The reaction scheme between DPPH free radicals and compound (2).

Figure 11

Optimized structure of comp.1and 2 by DFT. 


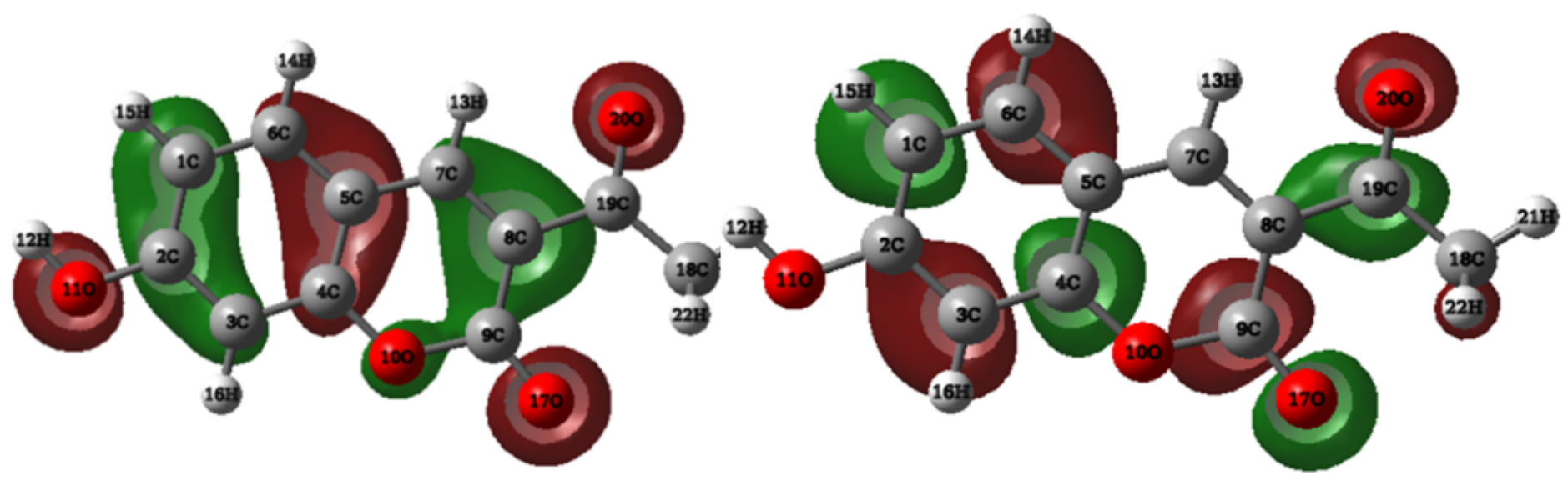

\section{HOMO}

\section{LUMO}

Figure 12

Atomic contributions in the HOMO, LUMO orbitals of Comp. 1.

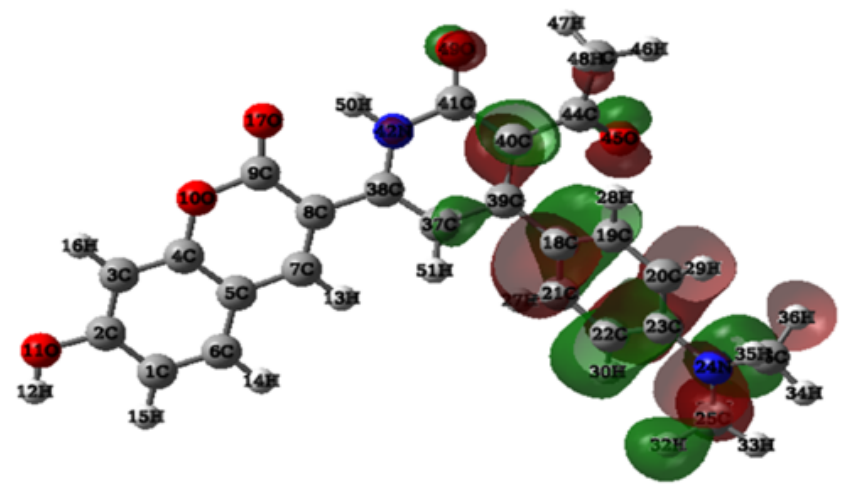

HOMO

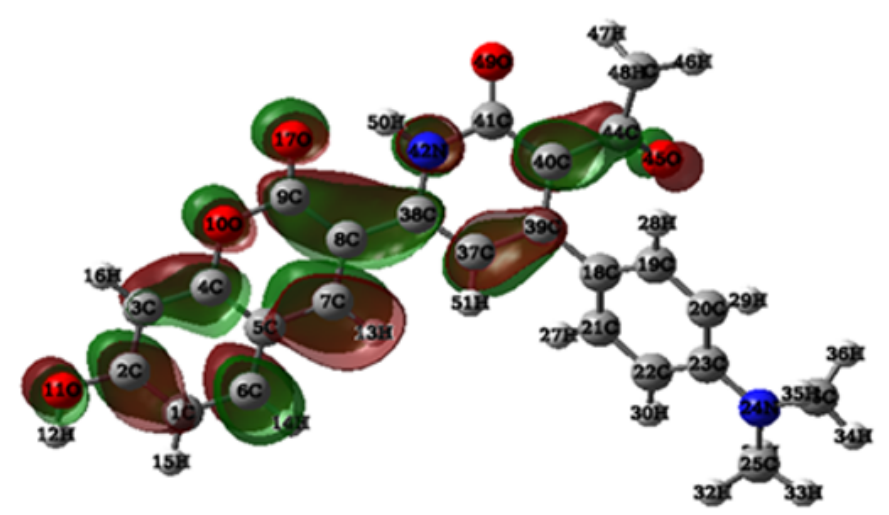

LUMO

Figure 13

Atomic contributions in the HOMO, LUMO, orbitals of Comp.2. 

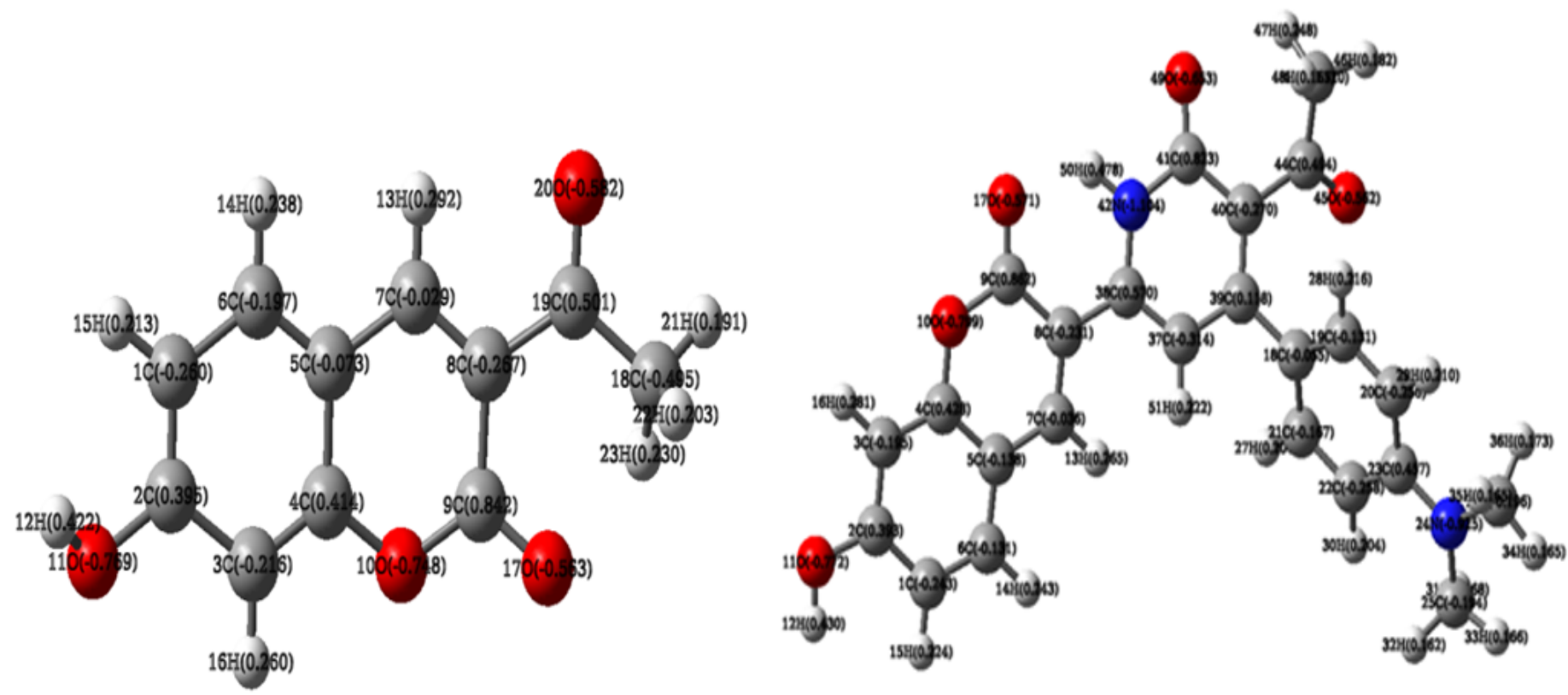

Figure 14

Optimized structure of comp.1 and 2 by DFT Atomic Charges and Stabilities. 